\title{
Некоторые аспекты развития САПР
}

\author{
М. Макушин ${ }^{1}$, В. Мартынов, д. т.н. ${ }^{2}$
}

УДК 658.512 | ВАК 05.13 .12

\author{
Системы автоматизированного проектирования - один из основных \\ инструментов, позволяющих превратить в реальные изделия результаты \\ перспективных НИОКР в области микроэлектроники. В то же время их \\ эволюция зависит от тенденций развития микро- и радиоэлектроники, \\ конечных рынков этой продукции. Все это стимулирует поставщиков \\ САПР осваивать новые подходы, расширять и углублять взаимодействие \\ с различными партнерами по всей цепочке поставок. Кроме того, существенно \\ расширяется использование облачных САПР.
}

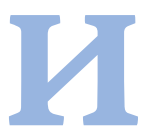

тоги 2019 года для полупроводниковых фирм оказались не очень радостными - они столкнулись со снижением доходов от продаж (до 418,3 млрд долл., что на 11,9\% меньше показателей 2018 года [1]) - особенно поставщики схем памяти. Но, с другой стороны, сегмент поставщиков инструментальных средств САПР пережил еще один год бурного роста. Это объясняется появлением новых технологий изготовления ИС, ни одна из которых еще не достигла стадии серийного производства, но уже требующих для дальнейшего развития новых и модернизированных средств САПР Кроме того, значительная часть роста доходов индустрии САПР связана с появлением все большего числа начатых разработок проектов создания ИС, реализуемых фирмами, не разрабатывавшими аппаратное обеспечение в прошлом. Предполагается, что существующие в этом плане тенденции сохраняются и сектор инструментальных средств САПР в ближайшие пять лет будет демонстрировать рост доходов в сложных процентах (CAGR) на уровне от 8 до $12 \%$.

На развитие рынка САПР оказывают влияние общая ситуация на рынке микро- и радиоэлектроники, появление и распространение перспективных конечных применений ИС, а также появление новых технологических разработок (новых технологий), определяющих процесс проектирования ИС.

\section{ОСНОВНЫЕ ФАКТОРЫ РАЗВИТИЯ САПР Рыночные факторы}

Трудно игнорировать стремительный рост средств искусственного интеллекта (ИИ) и его новыхприложений, реализуемых практически в каждом сегменте полупроводниковой промышленности, которая трансформируется под воздействием всплеска активности системных фирм,

Цнии «Электроника», главный специалист,

mmackushin@gmail.com.

ФГБНУ "Аналитический центр», проф., Минобрнауки РФ, эксперт. разрабатывающих заказные ИС. Корпорации Amazon, Facebook, Google, Microsoft и т.д. объявили о планах создания собственных ИС ИИ. При этом предпринимаются меры по стандартизации открытого интерфейса, определяемого открытой архитектурой представления предметной области (Open Domain-Specific Architecture, ODSA), которая может снизить сложность и стоимость проектирования высокопроизводительных ускорителей на основе лучших в своем роде чиплетов".

Подобная тенденция наблюдается не только в области ИИ. Так, автомобильные производители, такие как Tesla, Toyota (в сотрудничестве c Denso) и другие, самостоятельно начали НИОКР по разработке заказных ИС (или конструкций чиплетов) вместо того, чтобы обратиться, как обычно, к поставщикам первого уровня. Это, в свою очередь, также создает спрос на новые инструментальные средства САПР. При этом отмечается растущая важность функциональной безопасности, что подтверждается большим вниманием, которое уделяется стандарту ISO 26262. Так же высоки требования и в ряде других областей, где нет права на ошибку - таких как атомные электростанции, авионика, военные системы и имплантируемые медицинские устройства [2]

Кремниевые заводы наращивают свои возможности в производстве автомобильных ИС, готовясь к резкому скачку спроса на полупроводниковые приборы, используемые в перспективных системах помощи водителю (ADAS) и автономных транспортных средствах. Речь идет как о расширении функциональных возможностей

\footnotetext{
Chiplet - «чиплет», созданные в 2013 году в исследовательском центре Пало-Альто компании Хerox специализированные микросхемы, обладающие минимальной вычислительной мощностью и рядом других функций, позволяющие им стать малым микропроцессором, устройством хранения данных, сложной логической схемой или частью MEMS, выполняющих функции датчиков различных параметров, к примеру, освещенности, температуры, давления, движения и ускорения.
} 
традиционных автомобилей, так и о все бо́льшем распространении гибридных транспортных средств и электромобилей. Все основные поставщики САПР борются за расширение своих портфелей процессов, ориентированных на потребности клиентов, связанных с автомобильной промышленностью. Автомобильный сектор также привлекателен тем, что многие из используемых здесь полупроводниковых приборов не требуют при их изготовлении применения передовыхтехнологических процессов, а значит, на этом рынке может действовать большее количество поставщиков, прилагающих меньшие усилия.

Из-за возросших требований к безопасности и производительности неизбежной частью процесса производства микросхем стала виртуальная верификация конструкций. Процесс верификации включает в себя тестирование электрических и электронных компонентов при различных значениях температуры и давления. Растущая сложность автомобильной промышленности также способствует росту спроса на инструментальные средства САПР $[3,4]$.

Все эти тенденции стимулируют поставщиков САПР расширять сотрудничество с другими участниками цепочек поставок. Так, корпорация Synopsys присоединилась к консорциуму Autonomous Vehicle Computing Consortium (AVCC), целью которого является ускорение создания более безопасных и доступных автономных транспортных средств. Synopsys будет активно участвовать в разработке комплекса рекомендаций по системным архитектурам и вычислительным платформам; помогать определить эталонную архитектуру и платформу, отвечающие требованиям проектирования средств автономного вождения; предоставлять специализированные СФ-блоки, решения для проектирования и верификации, а также ПО [5].

Рынок инструментальных средств САПР сильно фрагментирован, и, в целом, конкурентное соперничество в индустрии инструментальных средств САПР остается высокИм. Одним Из основных подходов, который позволит минимизировать влияние конкурентного соперничества и поддерживать рентабельность, является концентрация (объединение). Соответственно, ожидается продолжение процесса слияний/поглощений, благодаря чему крупнейшие поставщики продолжат наращивать преимущества перед своими конкурентами.

К основным игрокам рынка САПР традиционно относят корпорации Mentor Graphics, Synopsis, IBM. Heкоторыми из наиболее значительных событий на рынке САПР в 2019 году можно считать работы Mentor Graphics и Synopsis. Первая заявила об успешном сотрудничестве входящей в нее группы Tanner EDA с корпорацией VPIphotonics (одним из первопроходцев в моделировании фотонных приборов), позволившим создать ряд динамичных и экономически эффективных решений анализа и проектирования для быстро развивающегося рынка кремниевой фотоники. Благодаря этому партнерству Mentor теперь использует более чем 20-летний опыт VPIphotonics для моделирования активных / пассивных/ гибридных фотонных ИС и систем. Вторая объявила об инвестициях в разработку решений в области ИИ, направленных на решение все более сложных задач проектирования приборов на уровне новейших технологических процессов с меньшими топологиями. Одна из реализаций Ии была направлена на расширение платформы проектирования Synopsys (Synopsys Design Platform), сокращение времени цикла достижения результатов (timeto-results, TTR), достижение новых уровней автоматизации (выполнение операций "одним нажатием кнопки») и установление новых стандартов качества результатов (QoR) при проектировании цифровых и заказных конструкций $[3,4]$.

\section{Технологические факторы}

Долгие годы с момента появления полупроводниковых приборов действовал так называемый закон Мура (эмпирическое наблюдение, а не природный/ физический закон - удвоение числа транзисторов на кристалле каждые 18 месяцев без увеличения удельной стоимости функций для конечного потребителя, сформулированное в конце 70-х годов 20 века Гордоном Муром, одним из основателей и ведущих специалистов корпорации Intel).

Однако в последние годы фундаментальные физические ограничения все больше затрудняют продолжение реализации этой тенденции, что в конечном итоге будет означать прекращение действия закона Мура. Ведущим фактором развития электронной промышленности становятся технологии больших данных (Big Data) и приложения ИИ, включая машинное обучение и глубокое обучение. Это привело к появлению гетерогенных вычислительных платформ - как традиционных систем-накристалле (SoC) со встроенными структурами вентильных матриц, программируемых пользователем (FPGA), так и массивных FPGA, которые также квалифицируются как SoC. Хорошим примером этой тенденции является 7-нм «Адаптивная платформа ускорения вычислений» (Adaptive Compute Acceleration Platform, ACAР) Versal корпорации Xilinx.

Масштабирование полупроводниковых технологических процессов в сторону меньших топологических норм продолжается, однако эта тенденция не всегда способна удовлетворить требования размещения на заданной площади большего числа транзисторов (из-за физических ограничений), необходимых для таких приложений, как ИИ. Соответственно, разработчики вынуждены искать другие решения, что, в свою очередь, приводит к предсказанному ранее "взрывному» росту численности конструкций 3D ИС. Практически каждая средняя или крупная полупроводниковая фирма, занимающаяся 
проектированием ИС, рассматривает многокристальные / многочиплетовые 3D ИС как альтернативу однокристальным монолитным ИС. Речь идет о развитии тенденции "Больше, чем Мур" в противовес тенденции "Больше Мура" . TSMC, крупнейший в мире кремниевый завод (контрактное производство ИС), и Samsung, один из крупнейших вертикально-интегрированных производителей ИС, использующий также модель кремниевого завода (автономное подразделение), в 2019 году объявили о создании базовых (эталонных - reference flow) технологических процессов создания 3D ИС. Этому решению предшествовала более чем десятилетняя история работ корпорации Cadence по переходу от моделирования процессов корпусирования, обеспечения целостности сигнала и мощности, корпусов с матричным расположением штырьковых выводов, поверхностного монтажа, систем-в-модуле (SiP) и методик 2,5D-корпусирования к 3D-корпусированию этажированных кристаллов. В настоящее время Cadence ускоряет сертификацию своих решений в области корпусирования совместно с TSMC, а в области многокристальной интеграции (multi-die integration, MDI) с компанией Samsung.

Это создает новые возможности для САПР - появляется необходимость в инструментальных средствах, реализующих 3D многокристальный / многочиплетовый подходы для планирования и управления формированием взаимосвязей и экспорта альтернативных конструкций / корпусов.

Некоторые из новыхтехнологий стимулируют развитие наиболее передовых технологических процессов, характеризующихся наименьшими топологическими нормами. В частности наблюдаются ускоренные темпы разработки технологических поколений с топологиями менее 10 нм, что обусловлено огромной потребностью в обработке данных. Значительный рост потребности в наращивании вычислительной мощности обусловлен быстрым внедрением облачных вычислений в нескольких вертикально организованных отраслях, включая высокопроизводительные вычисления (НРС или суперкомпьютеры), сети / средства связи 5G, искусственный интеллект, новейшие типы автомобильной электроники и мобильной техники.

\footnotetext{
* More than Moore - «Больше, чем Мур», заключающаяся в использовании 2,5-и 3-мерных архитектур, позволяющих существенно наращивать функциональность, сокращать занимаемое пространство и потребляемую мощность, а также в использовании перспективных материалов и приборныхструктур.

*:* More Moore - "Больше Мура», концепция, направленная на обеспечение дальнейшего действия так называемого закона Мура за счет новых материалов (углеродные нанотрубки, графен и т.д.) и приборных архитектур (молекулярная электроника, спинтроника и т.п.).
}

Развитие FPGA также во многом обусловлено потребностью в гибкости на новых рынках, таких как ИИ, автомобильная электроника и 5G-сети / средства связи, где алгоритмы, стандарты и интерфейсы продолжают изменяться быстрыми темпами. Решающее значение здесь имеет возможность программировать аппаратное обеспечение для учета различных изменений, но это не так просто, как кажется.

Преимущества FPGA в производительности и гибкости для приложений ИИ хорошо понятны. Самой значительной проблемой является модель программирования - она не может быть разрешена единым, универсальным способом. Она будет решаться комбинацией подходов, соответствующих экспертным знаниям разработчика.

Наблюдается увеличение использования FPGA - какавтономных, так и встроенных. Отмечается, что формальная проверка эквивалентности конструкций FРGА стала ключевой технологией для установления доверия в цепочке разработки. В ближайшее время ожидается значительный рост ее применения. Эта технология может гарантировать, что в процессе разработки в конструкцию не были введены никакие аппаратные трояны. Кроме того, инструментальные средства синтеза и размещения / трассировки FPGA оптимизируются, и все оптимизации должны быть проверены формально. Обе эти причины способствуют расширению использования проверки эквивалентности конструкций FPGA [2].

\section{РЫНОК САПР В ІІІ КВ. 2019 ГОДА}

По оценкам Альянса разработчиков САПР (Electronic System Design Alliance, ESD Alliance), сформированным на основе отчетных данных акционерных и частных фирмпоставщиков инструментальных средств САПР и полупроводниковых сложных функциональных (СФ) блоКОВ, в ІІІ КВ. 2019 года доходы отрасли выросли на 8,9\% по сравнению с III кв. 2018 года. Общая выручка за III кв. 2019 года составила 2,568 млрд долл. (рис. 1, табл. 1). С географической точки зрения большая часть доходов по-прежнему приходится на Америку (рис. 2, табл. 2).

В структуре доходов индустрии САПР почти 64\% доходов приходится собственно на системы проектирования, при этом наибольшие темпы прироста демонстрируются средствами проектирования печатных плат и многокристальных модулей. Немногим от них отстают темпы прироста средств проектирования ИС на физическом уровне и верификации (табл. 1). Темпы роста доходов от реализации СФ-блоков в последнее время замедлились, что не соответствует долгосрочным наблюдениям

\footnotetext{
*:* Place-and-route - размещение (компонентов) и трассировка (про водников, соединений), один из этапов проектирования многослойных печатных плат или микросхем в САПР электроники.
} 


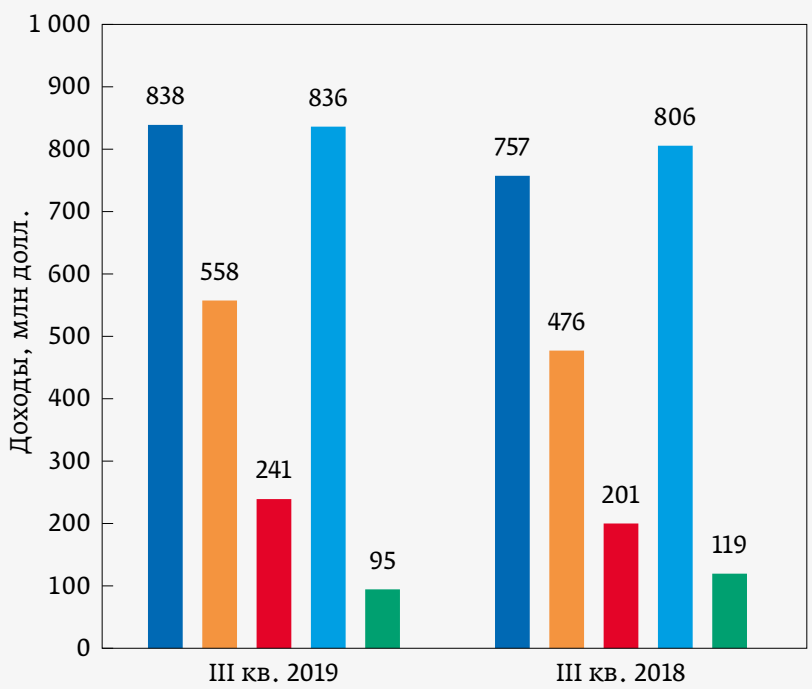

口 Средства автоматизированного проектирования Средства проектирования ИС на физическом уровне и верификации

- Средства проектирования печатных плат и многокристальных модулей

Доходы от разработки / реализации полупроводниковых СФ-блоков

- Услуги

Рис. 1. Структура доходов индустрии САПР в III кв. 2019 и 2018 годов

Действительно, с точки зрения долгосрочной перспективы наибольшие темпы прироста за период 1996-2019 годов наблюдались по доходам от разработки / реализации полупроводниковых СФ-блоков и по доходам от реализации средств проектирования ИС на физическом уровне и верификации (рис. 3) [6].

В конце 2018 года ESD Alliance объединил свои усилия с Международной организацией поставщиков оборудования и материалов для полупроводниковой

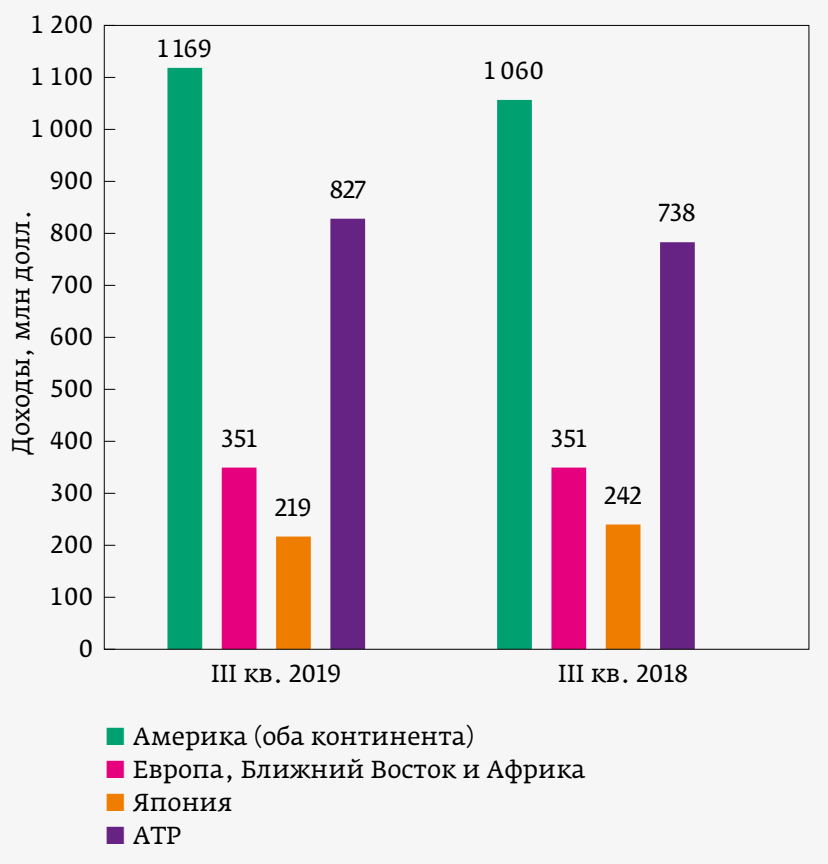

Рис. 2. Географическая структура доходов индустрии САПР в III кв. 2019 и 2018 годов

промышленности (SEMI). Цель состояла в том, чтобы интегрировать сегмент проектирования в цепочку поставок производства электроники, сформировав целостную экосистему. Отмечается, что у индустрии проектирования и полупроводниковой промышленности есть много общих проблем и узких мест, которые можно решить с помощью более широкого сотрудничества и партнерства.

\section{ПЕРСПЕКТИВЫ РЫНКА САПР}

В настоящее время больше половины ведущих поставщиков САПР поставляют как традиционные системы, так и системы для облачных применений. По данным фирм

Таблица 1. Структура доходов индустрии САПР за III кв. 2019 года

\begin{tabular}{|c|c|c|c|}
\hline Тип доходов индустрии САПР & $\begin{array}{c}\text { Объем доходов, } \\
\text { млн долл. }\end{array}$ & $\begin{array}{l}\text { Прирост, } \\
\text { \% }\end{array}$ & $\begin{array}{c}\text { Доля, } \\
\%\end{array}$ \\
\hline Доходы от реализации САПР, в том числе: & 1636,4 & 14,2 & 63,7 \\
\hline средства автоматизированного проектирования & 838,3 & 10,8 & 32,6 \\
\hline средства проектирования ИС на физическом уровне и верификации & 557,6 & 17,2 & 21,7 \\
\hline средства проектирования печатных плат и многокристальных модулей & 240,5 & 19,8 & 9,4 \\
\hline Доходы от разработки / реализации полупроводниковых СФ-блоков & 835,9 & 3,7 & 32,5 \\
\hline Доходы от оказания услуг & 95,4 & $-19,9$ & 3,8 \\
\hline Доходы индустрии САПР в целом & 2567,7 & 8,9 & 100 \\
\hline
\end{tabular}


Таблица 2. Географическая структура доходов индустрии САПР за III кв. 2019 года

\begin{tabular}{lccc} 
Страна / регион & $\begin{array}{c}\text { Объем доходов, } \\
\text { млн долл. }\end{array}$ & $\begin{array}{c}\text { Прирост, } \\
\%\end{array}$ & $\begin{array}{c}\text { Доля, } \\
\%\end{array}$ \\
Америка (оба континента) & 1169,9 & 10,4 & 45,6 \\
\hline АТР & 827,5 & 5,7 & 32,2 \\
\hline Европа, Ближний Восток и АФрика & 351,1 & 0,1 & 13,7 \\
\hline Япония & 219,3 & $-9,4$ & 8,5 \\
\hline Итого & $\mathbf{2 5 6 7 , 7}$ & $\mathbf{8 , 9}$ & $\mathbf{1 0 0}$ \\
\hline
\end{tabular}

Растущий спрос на современные электронные приборы со сложными конструкциями и необходимость уменьшения размеров ИС при одновременном повышении их производительности вынуждают производителей увеличивать инвестиции в НИОКР и внедрять все более совершенные инструментальные средства САПР. Поэтому ожидается, что спрос в этом секторе по сравнению с другими секторами будет высоким.
Absolute Reports и Kennet Research, это восемь корпораций и фирм с ограниченной ответственностью (табл. 3). К тому же три поставщика специализируются только на традиционных САПР, а еще четыре - исключительно на облачных САПР $[3,4,7]$.

По данным исследовательских фирм Absolute Reports и MarketWatch, мировой рынок инструментальных средств САПР, достигший в 2018 году 9,76 млрд долл., в 2024 году увеличится до 17,35 млрд долл., а среднегодовой прирост в сложных процентах (CAGR) за данный период составит 10,1\%. Инструментальные средства САПР в последние годы стали одним из основных факторов, определяющих темпы инновационного развития полупроводниковой промышленности.

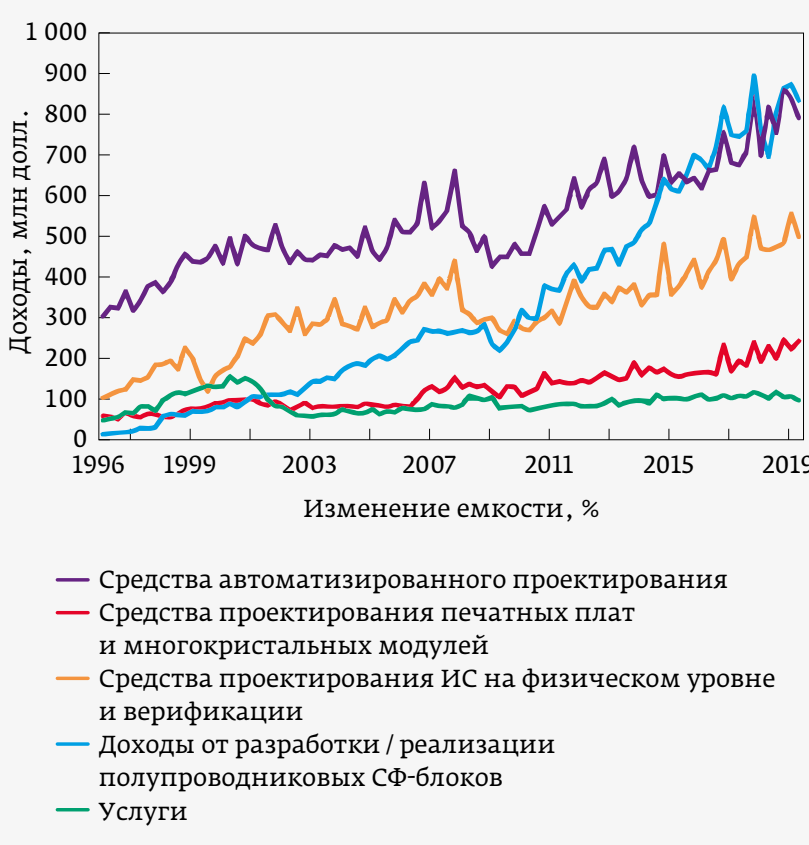

Рис. 3. Поквартальная (с I кв. 1996 г. по II кв. 2019 г.) структура доходов поставщиков инструментальных средств САПР

\section{Облачные САПР}

Одной из тенденций развития индустрии САПР является то, что их поставщики начали предлагать свои системы и ПО онлайн. Повсеместно растущее распространение Интернета облегчает потенциальным клиентам доступ к САПР (а также к библиотекам перспективных СФ-блоков и других элементов в качестве "строительных блоков" новых конструкций ИС) в сети/облаках и использование их для разработки и проектирования ИС. Следовательно, капитальные затраты фирм, использующих этот подход, сокращаются за счет снижения общей стоимости владения и затрат на инфраструктуру для размещения этих продуктов. Это также помогает улучшить управляемость цепочками поставок за счет значительного сокращения общего числа торговых посредников и дистрибьюторов, а также сократить время проектирования, уменьшить число ошибок и снизить объем затрат при производстве различных конечных систем [3, 4].

По данным фирмы Kennet Research, мировой рынок облачных САПР в 2018 году достиг 6,128 млрд долл., а в 2026 году превысит 8,92 млрд долл. при $\mathrm{CAGR}=5,51 \%$ за прогнозируемый период 2019-2026 годов. Рынок облачных САПР является рынком инструментальных программных средств, обычно используемых полупроводниковой промышленностью для проектирования сложных электронных систем. Инструментальные средства облачных САПР могут использоваться клиентами, расположенными в любых местах, помогая им разрабатывать и проектировать очень сложные конструкции схем, используемых в авиакосмической, автомобильной, военной, медицинской и других отраслях промышленности

Однако существуют различные факторы, которые в течение прогнозируемого периода будут препятствовать росту данного рынка. К ним можно отнести проблемы с драйверами облачных провайдеров, лицензирование инструментальных средств, информационную безопасность и т. п. [7]. 


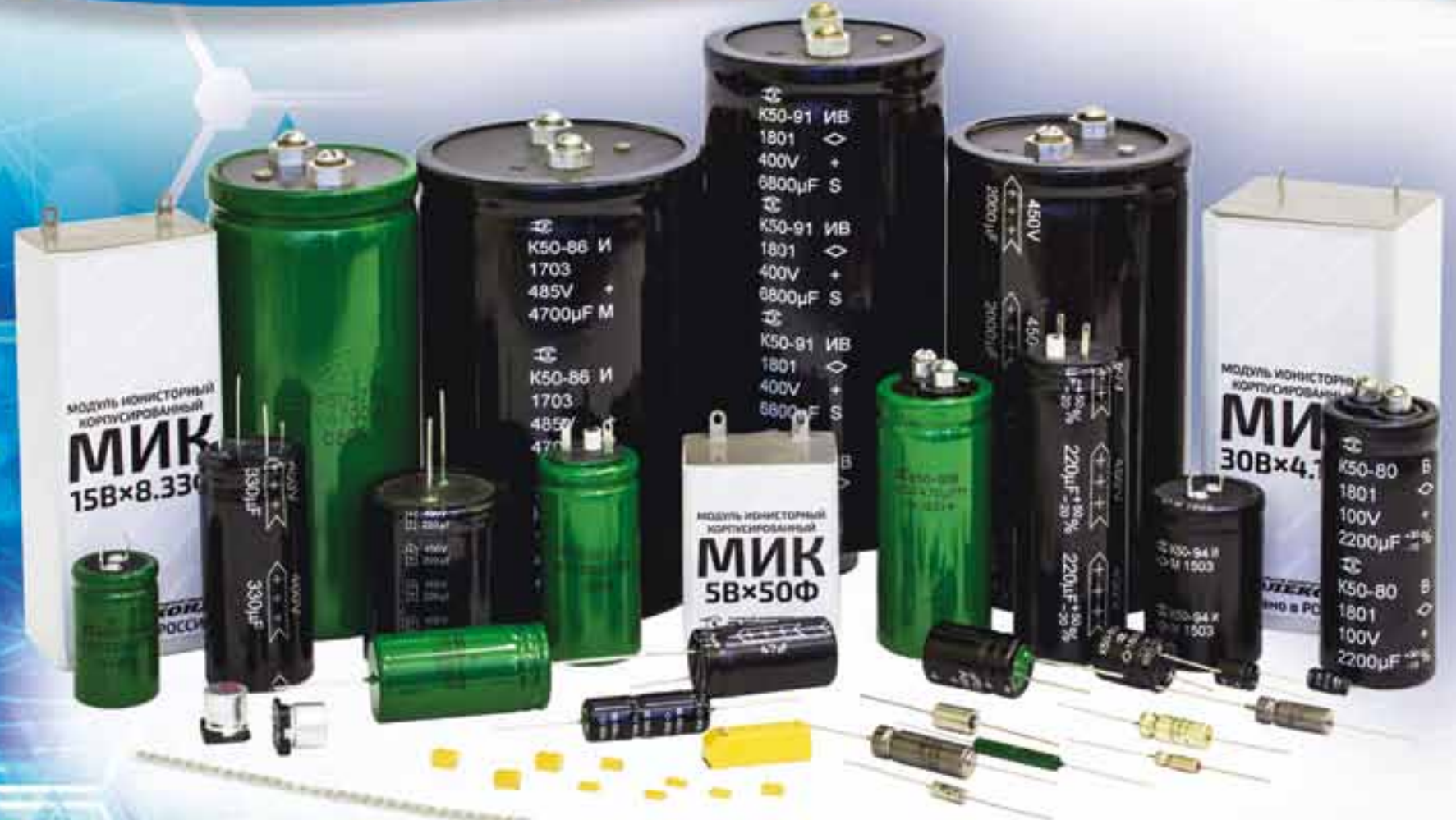

\section{Разработка и производство конденсаторов}

оксидно-электролитические алюминиевые конденсаторы К50-15, К50-17, К50-27, К50-37, К50-68, К50-74, К50-76, К50-77, К50-80, К50-81, К50-83, К50-84, К50-85, К50-86, К50-87, К50-88, К50-89, К50-90, К50-91, К50-92, К50-93, К50-94, К50-95(чип), К50-96, К50-98

объемно-пористые танталовые конденсаторы К52-1, К52-1М, К52-1БМ, К52-1Б, К52-9, К52-11, К52-17, К52-18, К52-19, К52-20, К52-21, К52-24, К52-26(чип), К52-27(чип)

оксидно-полупроводниковые танталовые конденсаторы

К53-1А, К53-7, К53-65(чип), К53-66, К53-68(чип), К53-71(чип), К53-72(чип), К53-74(чип), К53-77(чип), К53-78(чип)

суперконденсаторы (ионисторы) K58-26

накопители электрической энергии на основе модульной сборки суперконденсаторов

Система менеджмента качества сертифицирована на соответствие требованиям ISO 9001

Россия, 427968, Удмуртская Республика, г. Сарапул, ул. Калинина, 3 Тел.: (34147) 2-99-53, 2-99-89, 2-99-77, факс: (34147) 4-32-48, 4-27-53 e-mail: elecond-market@elcudm.ru, http://www.elecond.ru 
Таблица 3. Основные поставщики САПР

\section{Традиционные и облачные САПР}

Agnisys, Aldec, ANSYS, Cadence Design System, Mentor

Graphic (Siemens PLM Software), Synopsys, Xilinx, Zuken
Только традиционные САПР

Altium, Keysight Technologies,

Lauterbach
Только облачные САПР

Agilent, JEDA Technologies, MunEDA, Sigrity

\section{ВЛИЯНИЕ НОВЫХ ТЕХНОЛОГИЧЕСКИХ РАЗРАБОТОК НА ПРОЕКТИРОВАНИЕ ИС}

В октябре 2019 года журнал Semiconductor Engineering провел круглый стол о влиянии подходов, основанных на различных физических методах, и новых рыночных тенденций на проектирование ИС с представителями корпораций ANSYS, Xilinx, Infineon и Массачусетского технологического института (МТI). Отмечалось, что в современных приборах возникает много проблем, связанных с отводом тепла, динамической плотностью мощности, сопротивлением в разводке, утечкой тока из области памяти, шумовыми и электростатическими эффектами.

Имеющиеся в настоящее время технологии достаточно перспективны и могут развиваться дальше. В частности, это касается 3D ИС, но при этом необходимо совершенствовать системы моделирования и проектирования на физическом уровне, сохранять или даже снижать существующий допустимый коэффициент ошибок. Также необходимо реализовывать целостный подход к вопросам потребляемой мощности, быстродействию и отводу тепла при проектировании на технологических уровнях с минимальными топологиями. Эти трудности требуют дальнейшего совершенствования САПР и сопутствующих методологий. Например, одним из способов решения возникающих трудностей являются ИИ и машинное обучение. Кроме того, к важным факторам относится и соблюдение временного графика при завершении процесса проектирования и передаче в производство принципиально новых ИС, соответствующих рыночному спросу.

С 2009 года компания ANSYS при разработке CAПР большое внимание уделяет программируемой логике. Сегодня она использует лицензионные процессорные ядра фирмы ARM, технологию DDR дО3У, сетевые интерфейсы с расширенными возможностями, машинное обучение и т.д. - словом, продукты компании становятся все более высокотехнологичными (клиенты требуют). Это расширяет область применения продукции ANSYS и открывает новые горизонты в области 5G, автомобилестроения, машинного обучения и сетевыхтехнологий, казавшиеся невозможными еще 10 лет назад.

Идея создания универсальных кристаллов ИС, по мнению ANSYS, будет продолжать развиваться. В то же время много говорится про ИС для конкретных применений. Это имеет смысл для гигантских корпораций, создающих свои собственные ИС для конкретных случаев использования, и тесно связано с некоторыми из мегатенденций (автономное вождение и автомобильная сфера в целом, связь 5G, облачные вычисления, ЦОД и ИИ). Ведущие ученые-компьютерщики 30 лет назад были сосредоточены на работе в области САПР. Сегодня же многое в этой сфере так и осталось на уровне 1980-х годов, что влияет на успешность или неуспешность работы средств САПР в вычислительной среде. На передовом крае компьютерной науки за пределами индустрии моделирования САПР осуществляется множество важных инноваций, имеющих отношение к масштабированию вычислений. Но когда речь заходит о создании конкретной ИС, ограничением становится человеческий фактор, то есть системные принципы работы компаний входят в противоречие с работой отдельных сотрудников. У полупроводниковых фирм есть много возможностей добавить услуги, предоставляемые специализированными ИС, но эти возможности не безграничны. Если у компании есть всего один ведущий разработчик, то имеющиеся возможности в области ИИ и машинного обучения применительно К САПР помогут повысить эффективность работы младших специалистов, и здесь открываются долгосрочные перспективы. Использование ИИ и машинного обучения в сочетании со средствами проектирования должно помогать выявлять возникающие проблемы и исправлять их.

ANSYS отмечает рост числа случаев повторной сборки ИС после исправления проблем. При этом в наиболее критичных областях обнаруживается, что нельзя исправить то, что невозможно измерить. Используемые методологии на основе допусков также влияют на стоимость, но они весьма консервативны. Что касается параметров напряжения и синхронизации, осведомленность разработчиков выше, при этом тренд цены и объема (price-volume trend, PVT), изменчивость процесса, изменчивость напряжения и температура могут быть смоделированы гораздо лучше. Сбои синхронизации напряжения реальны, но есть возможность улучшить ситуацию. Гораздо сложнее обстоят дела с электромагнитными явлениями на кристалле ИС. Теперь, когда существуют встраиваемые SERDES (параллельнопоследовательные и последовательно-параллельные преобразователи) и процессоры цифровой обработки сигнала с высокой пропускной способностью, эффекты индуктивности на кристалле ИС становятся крайне разрушительными. Это огромная проблема для разработчиков - число повторных сборок растет, и это связано 
с тем, что используются различные физические взаимодействия, которые необходимо как можно лучше моделировать.

Infineon, как и ряд других фирм, отказалась от дальнейшего масштабирования в пользу совершенствования 22-нм технологии (обеспечение большей безопасности и энергоэффективности) под нужды автомобильной промышленности. Ее специфические требования создают дополнительные сложности - для Infineon это вопросы интеграции, отладки и устранения неполадок. С точки зрения аналитики в разных областях требуются свои подходы и нужны способы их одновременного быстрого просмотра и анализа. Налицо фундаментальные изменения: если раньше новейшие микросхемы с наименьшими топологиями использовались в смартфонах или ПК, то сегодня, благодаря внедрению ИИ, распространению 5G и краевых вычислений и т.д., происходит процесс переоценки ценностей. Все это влияет на проектирование.

Infineon создает много готовых систем для дальнейшей доработки под конкретного пользователя. Большие достижения наблюдаются в сфере систем-в-модуле и этажерок кристаллов ИС, при этом во весь рост встают вопросы синхронизации и совместимости различных областей применения ИС, как и проблемы надежности, в том числе связанные с высокой рабочей температурой. Кроме того, нужно обращать внимание на энергоэффективность: в целях экономии энергии на работающем кристалле ИС должна быть возможность отключения одной, двух или трех зон. Современное проектирование ИС в гораздо большей степени связано с конкретными конечными применениями, чем раньше. В прошлом основное внимание при создании новых ИС уделялось соответствию наименьшим проектным нормам из возможных. Теперь на рынке появляется все больше новых продуктов, основанных на знании потребностей конкретной области деятельности.

Infineon утверждает, что часто для получения 90\%-ной базовой функциональности, прежде чем она будет физически реализована в структурах ИС, необходимо планировать действия на два шага вперед. Иными словами, растет важность предварительного моделирования. Спецификации всегда меняются в зависимости от требований конкретного клиента.

Корпорация Xilinx (один их крупнейших поставщиков как FPGA, так и (АПР) считает, что не существует универсальной FРGА для всех рынков - некоторые комплектующие должны быть специализированными, учитывающими потребности разных сегментов рынка. Для сетей 5G подходит одно, для автомобильной промышленности другое. Это верно и для специализированных ИС (ASIC), ожидается, что сегментация этого рынка будет продолжаться. В то же время компаниям, выводящим на рынок несколько ИС вместо одной, приходится решать и больше проблем. Одно из самых интересных изменений, наблюдаемых сейчас в области вычислений, - переход от вертикального масштабирования к горизонтальному. Для многих подходов, используемых сегодня, требуются отдельные системы и серверы, зачастую с максимально возможной емкостью и производительностью. Однако всему есть предел. Дальнейший путь - это горизонтальное масштабирование, возможность производить гибкие вычисления в "облаке» и наращивать число используемых серверов при необходимости достижения большей емкости ресурсов. Этот подход требует непрерывного анализа создаваемых систем с точки зрения размера, и это проблема не только полупроводниковой, но и электронной промышленности в целом.

MTI предлагает взглянуть на проблемы универсальных и специализированных ИС (ASIC) в более широких масштабах развития экосистемы. По всей видимости, наблюдается повторение истории развития специализированных микросхем или аппаратного оборудования в целом. Так, например, процессоры цифровой обработки сигнала превратились в процессоры обработки речи или процессоры видеосистем, и они становятся все более универсальными. Наблюдаются волнообразные всплески инноваций в очень узких областях использования, а затем идет процесс универсализации. То же самое происходит сейчас с ИС ИИ, которые переходят от очень специализированных решений к более универсальным процессорам, способным решать широкий круг проблем.

МТI в течение многих лет занимался разработками ИС, готовых для внедрения в производство. Очевидно, что очень многое зависит от условий работы, от взаимодействия с окружающей обстановкой, от сферы конечного применения и т.д. Таким образом, правильно подобрать конструкцию ИС, которая будет отвечать всем требованиям при первом использовании, становится все сложнее. Современные методики проектирования помогают в этом, но решающее значение имеет изучение всех физических взаимодействий внутри кристалла ИС. Проектировщикам необходимо собирать все возможные данные, строить модели (в том числе физического уровня), а также использовать все эти результаты в моделях машинного обучения. Необходимо как можно быстрее вносить изменения в конструкцию (если это возможно), но не пытаться решить все проблемы сразу [8].

$$
\because *
$$

Индустрия САПР отвечает за разработку инструментальных средств, позволяющих осуществлять процесс проектирования ИС при уровне затрат, обеспечивающем рентабельность. Ее дальнейшие перспективы неразрывно 
связаны с тенденциями развития поставщиков материалов и оборудования для полупроводниковой промышленности, производителей изделий микро- и радиоэлектроники, конечных рынков этих изделий. От поставщиков САПР требуется дальнейшее повышение уровня автоматизации проектирования и сокращения его сроков за счет использования средств ИИ, методик машинного обучения, повышения вычислительной мощности, использования методов массового параллелизма.

\section{ЛИТЕРАТУРА}

1. Davis Sh. Worldwide Semiconductor Revenue

Declined 11,9\% in 2019 // Semiconductor Digest Daily. January 17. 2020

https://www.semiconductor-digest.com/2020/01/17/ worldwide-semiconductor-revenue-declined-11-9-in-2019/

2. Bailey Br. What Worked, What Didn"t In 2019// Semiconductor Engineering. December $19^{\text {th }} .2019$. https://semiengineering.com/what-worked-whatdidnt-in-2019/

3. Electronic Design Automation Tools (EDA) Market - Growth, Trends, and Forecast (2019-2024) // Absolute Reports. 2019 https://www.absolutereports.com/electronic-designautomation-tools-eda-market-13999439
4. Electronic Design Automation Tools (EDA) Market 2019: Comprehensive Analysis on Business Summary, Product Opportunity and Current Industry Development 2024. Nov 11. 2019

https://www.marketwatch.com/press-release/electronicdesign-automation-tools-eda-market-2019-comprehensiveanalysis-on-business-summary-product-opportunity-andcurrent-industry-development-2024-2019-11-11

5. Davis Sh. Synopsys Joins New Autonomous Vehicle Computing Consortium // Semiconductor Digest Daily. January 17. 2020

https://www.semiconductor-digest.com/2020/01/17/ synopsys-joins-new-autonomous-vehicle-computingconsortium/

6. MMS Newsletter. Third Quarter 2019. Electronic System Design Alliance. Q3. 2019 http://esd-alliance.org/wp-content/ uploads/Newsletters/mss/MSs_Newsletter.pdf

7. Global Cloud Electronic Design Automation (EDA) Market Size study and Regional Forecasts 2019-2026. Kennet Research, Jul 2019 https://www.kennethresearch.com/report-details/ cloud-electronic-design-automation-eda-market/10085866

8. Sperling E. Less Margin, More Respins, And New Markets // Semiconductor Engineering. 10.10. 2019 https://semiengineering.com/less-margin-more-respins-andnew-markets/(Endnotes)

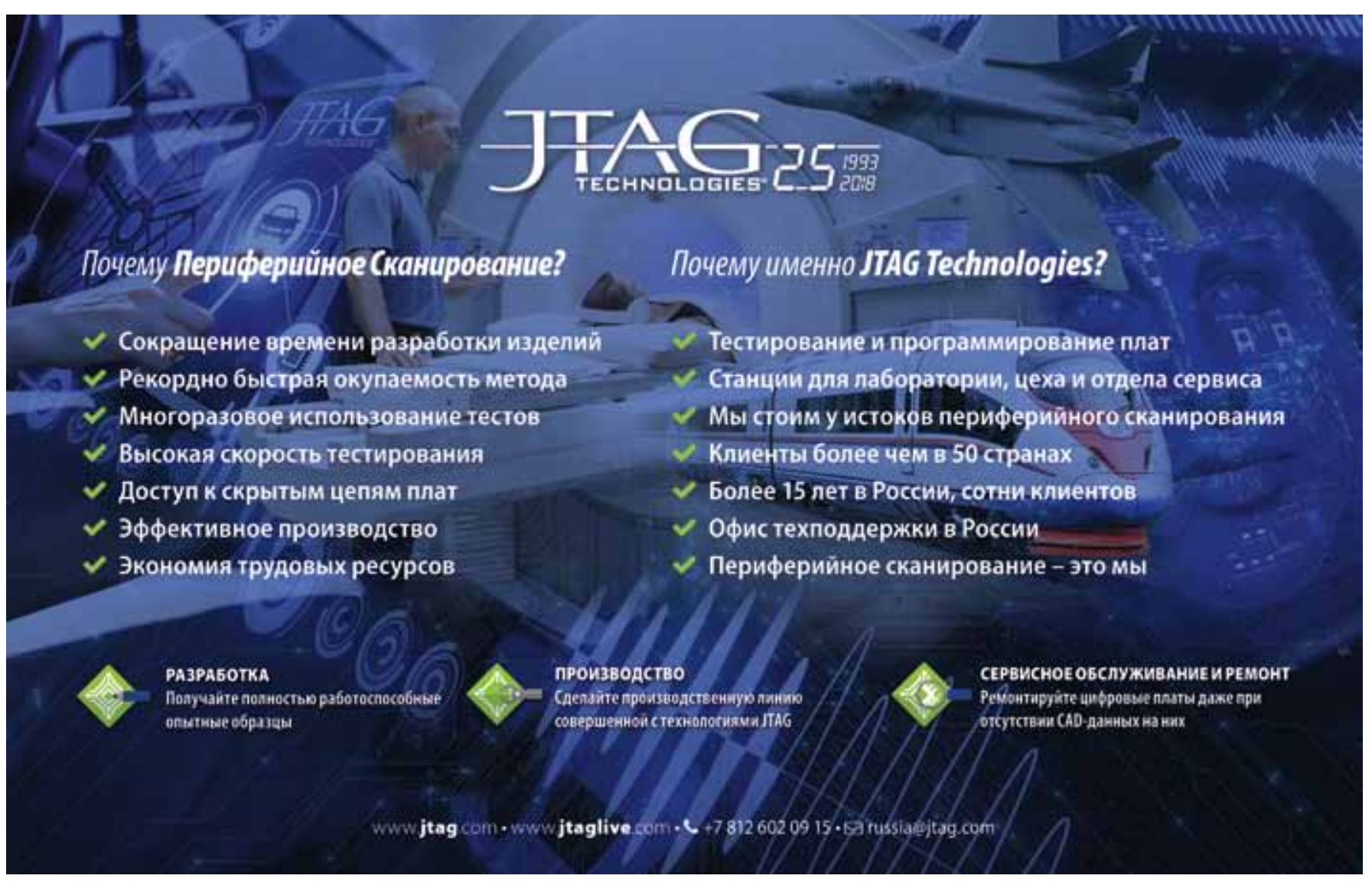

